\title{
Gas Permeability Change With Deformation and Cracking of a Sandstone Under Triaxial Compressiongas Permeability Change With Deformation and Cracking of a Sandstone Under Triaxial Compression
}

\author{
Yuan-Jian LIN
}

China University of Mining and Technology

Jiang-Feng LIU ( $\nabla$ jeafliu@hotmail.com )

China University of Mining and Technology https://orcid.org/0000-0002-4847-7102

\section{Tao CHEN}

Sinopec Shengli Oilfield Oil Drilling Technology Research Institute

\section{Bing-Xiang HUANG}

China University of Mining and Technology

Kundwa Marie Judith

China University of Mining and Technology

Hai-Bo BAI

China University of Mining and Technology

\section{Research Article}

Keywords: sandstone, permeability, pore/fracture structure, deformation, gas pressure

Posted Date: January 7th, 2022

DOI: https://doi.org/10.21203/rs.3.rs-1085538/v1

License: (c) (i) This work is licensed under a Creative Commons Attribution 4.0 International License. Read Full License 


\section{Gas Permeability Change with Deformation and Cracking of a Sandstone under Triaxial Compression}

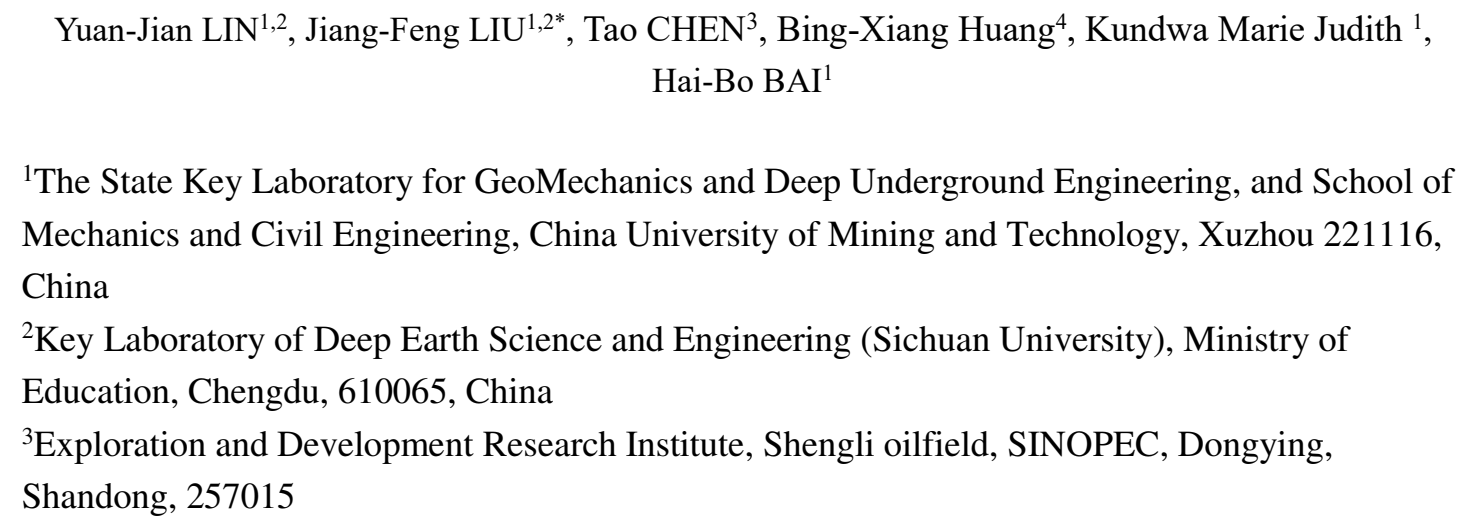


Abstract:

In this paper, a THMC (Thermal-Hydrological-Mechanical-Chemical) multi-field coupling triaxial cell was used to systematically study the evolution of gas permeability and the deformation characteristics of sandstone. The effects of confining pressure, axial pressure, and air pressure on gas permeability characteristics were fully considered in the test. The gas permeability of sandstone decreases with increasing confining pressure. When the confining pressure is low, the variation of gas permeability is greater than the variation of gas permeability at high confining pressure. The gas injection pressure has a significant effect on the gas permeability evolution of sandstone. As the gas injection pressure increases, the gas permeability of sandstone tends to decrease. At the same confining pressure, the gas permeability of the sample during the unloading path is less than the gas permeability of the sample in the loading path. When axial pressure is applied, it has a significant influence on the permeability evolution of sandstone. When the axial pressure is less than $30 \mathrm{MPa}$, the gas permeability of the sandstone increases as the axial pressure increases. At axial pressures greater than $30 \mathrm{MPa}$, the permeability decreases as the axial pressure increases. Finally, the micro-pore/fracture structure of the sample after the gas permeability test was observed using 3D X-ray CT imaging.

Keywords: sandstone; permeability; pore/fracture structure; deformation; gas pressure

\section{Introduction}

Natural gas is considered to be a cleaner and more efficient energy source than oil and coal. Unconventional resources, such as tight sandstone gas, are at the bottom of the global natural gas resource pyramid. However, its potential resources are considerably greater than the conventional natural gas reserves (Khlaifat et al., 2011). At present, although the exploitation of tight sandstone gas is still in its early stages, its reserves are relatively large, with recoverable reserves of approximately $1 / 3$ of the total amount of natural gas. Sandstone gas is a typical unconventional natural gas resource stored in low-permeability or ultra-low-permeability tight sandstone reservoirs. Sandstone gas is difficult to mine by conventional techniques. Therefore, it is usually necessary to produce natural gas of economic value through large-scale fracturing or special gas recovery process technology (Figure 1). Reservoir porosity and permeability are two important parameters for reservoir evaluation(Wang et al., 2017a; Xiao et al., 2017; Yang and Huang, 2020). In particular, the stress sensitivity of gas permeability strongly impacts the mining effect of low permeability tight oil and gas fields.

In recent years, extensive research has been conducted on the gas permeability characteristics of sandstone(Amann-Hildenbrand et al., 2016; Duan et al., 2014; Fu et al., 2015; Ghanbarian 
et al., 2016; Wang et al., 2017b; Wang and Cheng., 2020; Wu et al., 2020; Zhang et al., 2019).

Results indicate that crack closure primarily causes the reduction in the effective permeability of the gas due to the increase in the confining pressure under the condition of constant water content. In addition, studies have also found that some samples even have a large dispersion of initial effective permeability. However, there is a confining pressure-saturation threshold, and when this threshold is exceeded, the effective gas permeability of the samples decreases abruptly (Duan et al., 2014; Fu et al., 2015; Wang et al., 2017b). However, these studies have neglected the evolution of gas permeability after sample fracture. In mining, the gas permeability of sandstone after fracturing is a highly important parameter that cannot be ignored. In addition, the microscopic pore structure after fracturing is of great significance for evaluating the mesoscopic mechanism of gas migration. At present, there is little research on this aspect.
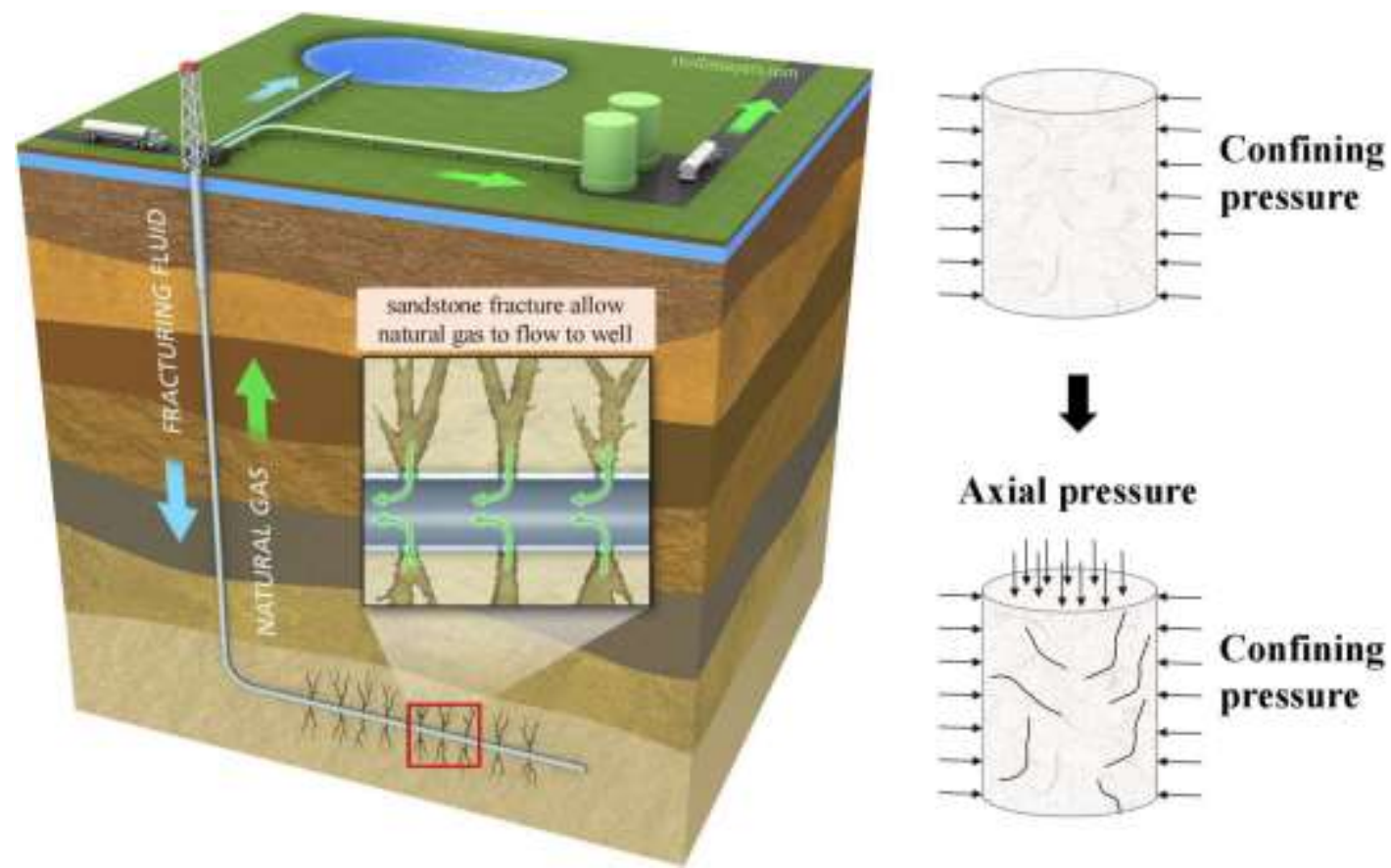

Axial pressure

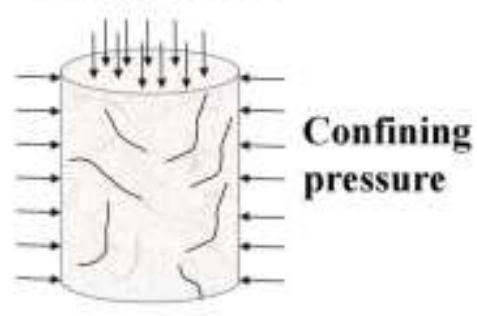

Figure 1. Schematic diagram of sandstone gas mining (modified from www.studiosayers.com).

In this paper, we focused on the evolution of gas permeability during cyclic loading and unloading and considered the effect of air pressure on gas permeability characteristics. Furthermore, we also studied the evolution law of gas permeation of sandstone during fracturing and reconstructed the fractured specimen by $\mathrm{CT}$ scanning to observe the internal pore fracture structure.

\section{Materials and methods}

\subsection{Materials and experimental scheme}

The samples were obtained from a field in Anhui Province, China. The samples were prepared as cylinders $50 \mathrm{~mm}$ in diameter and $20 \mathrm{~mm}$ in length. Two samples were first placed into a 
110 triaxial cell to perform gas permeability tests. At the end of the test, one of the specimens was

111 sent for three-dimensional CT scanning to observe the internal cracks.

112 Confining pressure was applied from $1 \mathrm{MPa}$ until $20 \mathrm{MPa}$. 0.5 MPa was chosen as an initial 113 gas injection pressure to avoid affect the pore structure. Different air pressures were applied at 114 different confining pressure stages. The purpose was to study the barometric sensitivity of 115 permeability. In the first phase, the confining pressure is loaded and unloaded in a stepwise 116 manner. To consider the complexity of the stress on the sand in the field, we performed two 117 cycles of loading and unloading. In the second stage, the confining pressure was restored to 20 $118 \mathrm{MPa}$, and axial pressure was applied. The axial pressure was applied from $5 \mathrm{MPa}$ with stepwise 119 loading (loading gradient of $5 \mathrm{MPa}$ ) until the sample was fractured. The gas pressure was 120 maintained at $0.5 \mathrm{MPa}$ throughout the loading process. The flow chart of the experimental 121 scheme is shown in Figure 2.

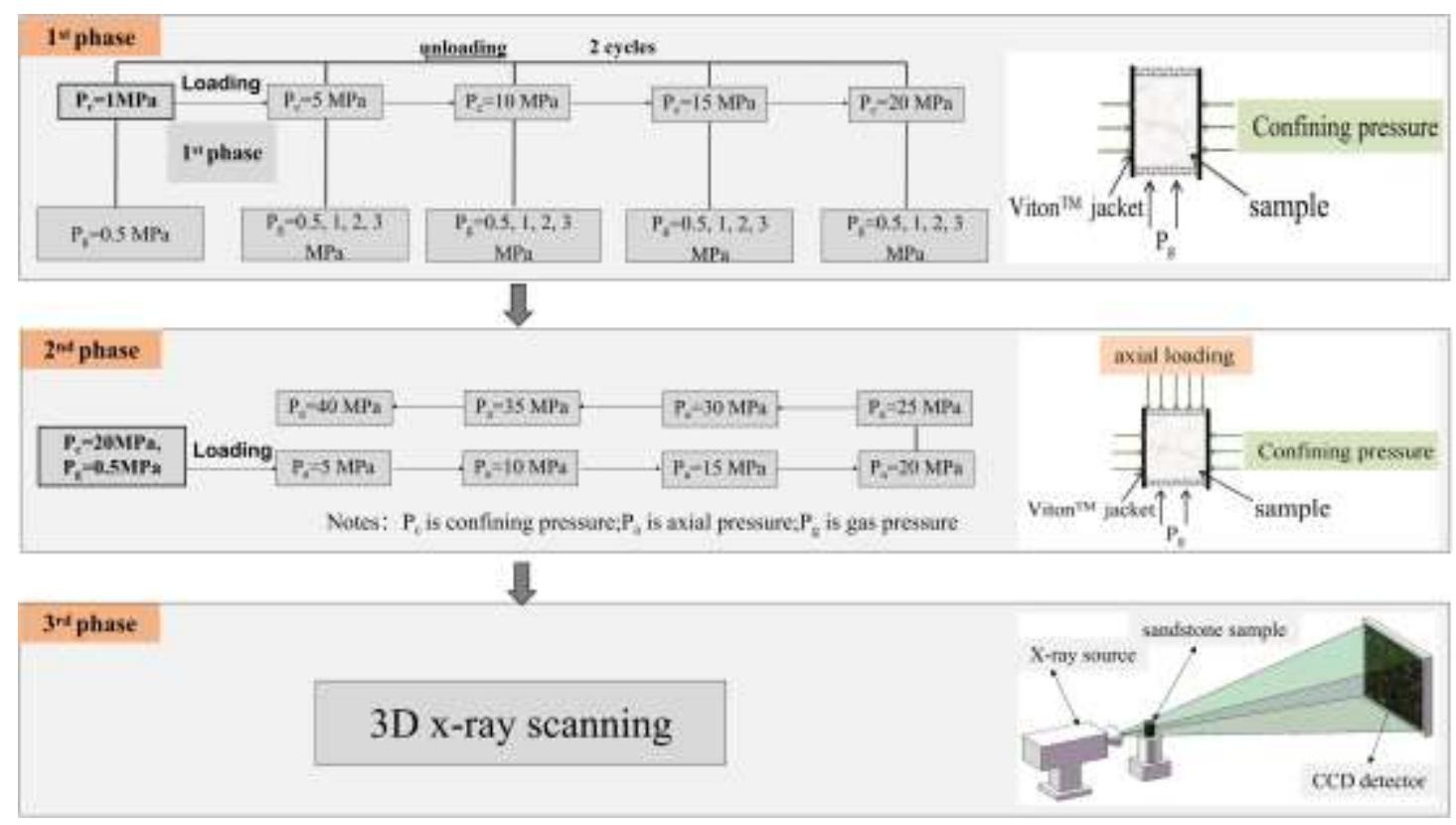

Figure 2. Experimental scheme.

1242.2 Gas permeability calculation method

125 The permeability calculation was performed using the steady-state method, which works well 126 for samples with a permeability higher than $10^{-19} \mathrm{~m}^{2}$ and has been used widely in many 127 studies(Liu et al., 2020; Liu et al., 2015). Another reason is that we need to carry out 128 Klinkenberg (or slip) experiments, and the steady-state method is easier to implement. This 129 study also applied this method combined with our gas permeability test device (Figure 3 ). The 130 servo system controls the confining pressure/axial pressure. To ensure safety, Argon was 131 chosen and injected from the gas source into a set volume cylinder $(0.4 \mathrm{ml})$. The gas was later 132 injected into a triaxial pressure chamber at a given pressure $P_{1}$, and the gas pressure drop $\Delta P$ 133 was recorded over time $\Delta t$. The average pressure $\left(\mathrm{P}_{\text {mean }}\right)$ in the cylinder during $\Delta t$ is 
$\mathrm{P}_{\text {mean }}=P_{1}-\Delta P / 2$. Darcy's law states that the formula for determining the effective gas

135 permeability (k eff) is as follows:

$$
k_{e f f}=\frac{\mu_{g} V_{0}}{A} \frac{2 h \Delta P}{\left(P_{m e a n}^{2}-P_{0}^{2}\right) \Delta t}
$$

where $\mu_{g}$ is the fluid's dynamic viscosity $\left(2.2 \times 10^{5} \mathrm{~Pa} \cdot \mathrm{s}\right), V_{0}$ is buffer reservoir's volume,

$138 h$ is the sample height, and $A$ is the sample's upper/bottom surface area.

\section{9}

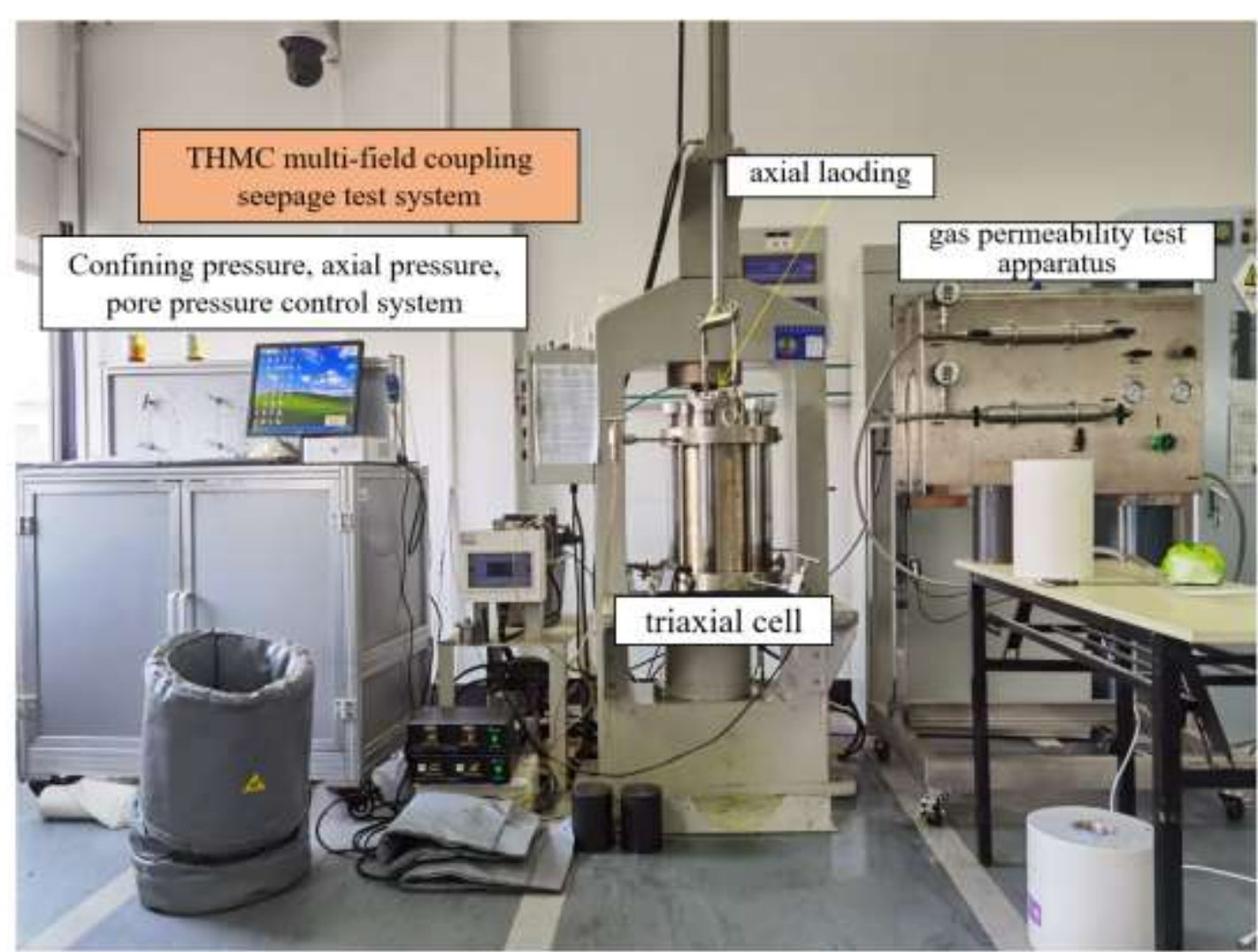

Figure 3. Gas permeability measurement devices.

2.3 Klinkenberg (or slip) effect

The Klinkenberg (or slip) effect refers to the phenomenon where the gas exhibits a finite velocity on the solid wall, and the velocity of the liquid is zero when the gas moves through the porous medium. This phenomenon exhibited by the gas is known as the slip or the Klinkenberg effect (Klinkenberg, 1941; Wang et al., 2018):

$$
k_{\text {eff }}=k_{\text {int }}\left(1+\frac{4 c l}{r}\right)=k_{\text {int }}\left(1+\frac{b}{P}\right)
$$


Where:

$151 K_{\text {int }}$ is the intrinsic permeability; $l$ is the average free path of the gas molecule; $r$ is the pore 152 radius; $c$ is the scale factor; $b$ is the Klinkenberg coefficient or the slip factor, and $P$ is the pore 153 pressure. The slip factor is defined as

$b=\frac{c \kappa T}{\sqrt{2} \pi r^{3}}$

155 where $\kappa$ is the Boltzmann constant, and $T$ is the absolute temperature.

156 2.4 Micro-CT observation test

157 The three-dimensional scan was tested in the Advanced Analysis \& Computation Center, China

158 University of Mining and Technology. The test instrument is Carl Zeiss Xradia 510 Versa High

159 Solution 3D X-ray Microanalyser, with a 3D spatial resolution: $55 \mu \mathrm{m}-0.5 \mu \mathrm{m}$. To observe the

160 macroscopic crack of the rock sample, the observation was performed after the triaxial

161 compression test.

\section{Results and discussion}

\section{$163 \quad 3.1$ Effect of loading/unloading on gas permeability}

164 Figures. 4(a) (b) shows the evolution of gas permeability of two samples (C1 and C2) under

165 different confining pressures. To avoid the interference of air pressure on the permeability

166 results, we only plotted the gas permeability results with a pressure of $0.5 \mathrm{MPa}$. Throughout

167 the experiment, gas permeability was reduced by order of magnitude (from $1.48 \times 10^{-15} \mathrm{~m}^{2}$ to

$1681.39 \times 10^{-16} \mathrm{~m}^{2}$ ) for sample $\mathrm{C} 1$ and by 3.3 times (from $5.01 \times 10^{-16} \mathrm{~m}^{2}$ to $1.50 \times 10^{-16} \mathrm{~m}^{2}$ ) for sample

169 C2. The gas permeability decreases with increasing confining pressure. When the confining 170 pressure is less than $5 \mathrm{MPa}$, gas permeability decreases rapidly: 7.59 times for sample $\mathrm{C} 1$ and 1712.27 times for $\mathrm{C} 2(0 \rightarrow 5 \mathrm{MPa})$. When the confining pressure is greater than $5 \mathrm{MPa}$, the rate of 172 decrease of gas permeability gradually reduces 1.40 times for sample C-1 and 1.66 times for

$173 \mathrm{C} 2(5 \rightarrow 20 \mathrm{MPa})$. When considering the explanation for this phenomenon, there are some large 174 pores inside the sample at the initial stage. As the confining pressure increases, this part of the 175 pores closes rapidly, and the permeability decreases sharply. As the confining pressure 176 continues to increase, the remaining pores are less sensitive to the confining pressure. 177 Therefore, the change in confining pressure has a small effect on the permeability.

178 For the unloading stage: $20 \rightarrow 5 \mathrm{MPa}$, the gas permeability difference between the unloading 179 stage and loading stage under the same confining pressure is small (e.g., sample $\mathrm{C} 1: 1.50 \times 10^{-}$ $180{ }^{16} \mathrm{~m}^{2}$ (unloading -10MPa) vs $1.62 \times 10^{-16} \mathrm{~m}^{2}$ (loading-10MPa)). When the confining pressure is 181 decreased from $5 \mathrm{MPa}$ to $1 \mathrm{MPa}$, the hysteresis effect of the permeability is obvious. The 182 permeability corresponding to the unloading stage under the same confining pressure is much 
183 smaller than the permeability corresponding to the loading stage (e.g., sample $\mathrm{C} 1: 5.51 \times 10^{-16}$

$184 \mathrm{~m}^{2}$ (unloading-1MPa) vs $14.81 \times 10^{-16} \mathrm{~m}^{2}$ (loading-1MPa)).

185 For the second cycle of loading and unloading, the test results in the loading phase are mostly

186 the same as the results in the unloading phase of the first cycle. Gas permeability changes very

187 little throughout the unloading stage (compared to results from other stages under the same

188 confining pressure). When the confining pressure returned to the initial value (1 MPa), the gas

189 permeability was slightly less than the loading phase (sample $\mathrm{C} 1: 5.51 \times 10^{-16} \mathrm{~m}^{2} v s .4 .07 \times 10^{-}$

$190{ }^{16} \mathrm{~m}^{2}$ ). Compared with the first cycle loading phase results, the gas permeability decreased from

$19114.81 \times 10^{-16} \mathrm{~m}^{2}$ to $4.07 \times 10^{-16} \mathrm{~m}^{2}$. This indicates that some pores cannot be opened after being

192 closed, although the confining pressure returns to the initial state. A similar trend was found for 193 sample C2.

194

195

196

197

198

199

200

201

202

203

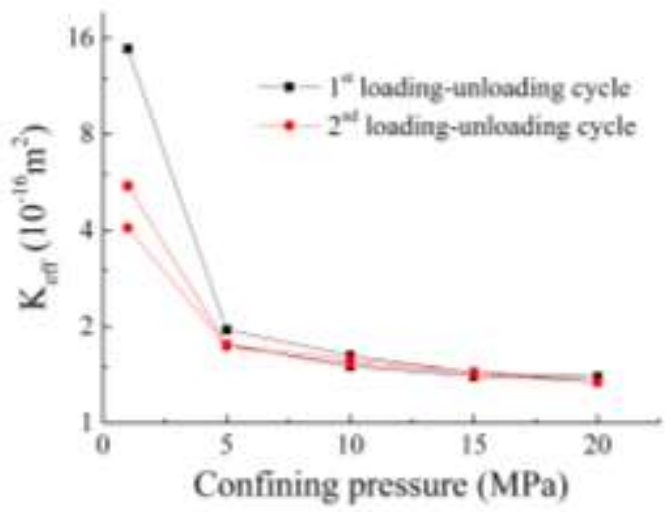

(a)

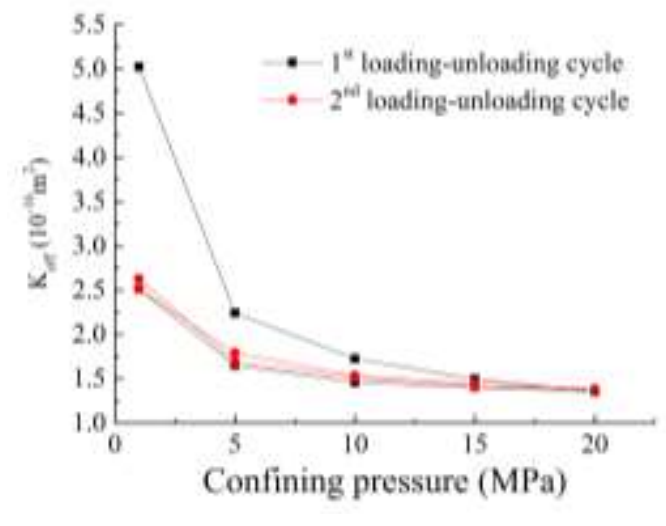

(b)

Figure 4. Effect of confining pressure on gas permeability of (a) sample C-1 and (b) C-2;

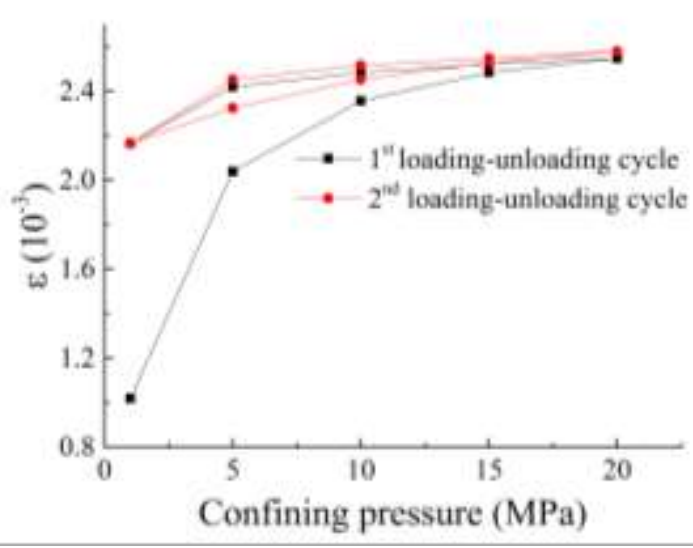

(a)

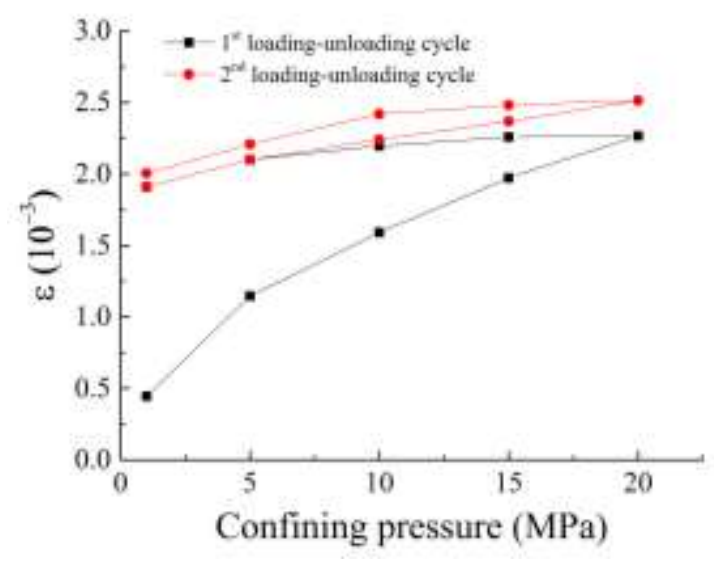

(b)

Figure 5. Effect of confining pressure on deformation of sandstone $\left(\mathrm{P}_{\mathrm{g}}=0.5 \mathrm{MPa}\right)$ (a) sample $\mathrm{C}-1$ and (b) sample C-2.

Figures. 5(a) (b) shows the deformation characteristics of the sample during the gas permeability test. In general, the deformation law follows the evolution law of gas permeability. Similarly, when the confining pressure is less than $5 \mathrm{MPa}$, the deformation of the sample is 
large. Above $5 \mathrm{MPa}$, the deformation of the sample is less sensitive to the confining pressure.

205 In the second loading and unloading cycle, the deformation of the sample is mainly in the elastic

206 range, and some deformation is unrecoverable compared with the first cycle.

\section{$207 \quad 3.2$ Effect of gas pressure on gas permeability}

208 Figures 6(a) (b) show the effect of gas pressure on gas permeability during the first and second 209 loading-unloading cycles. First, the gas permeability decreases with increasing gas pressure at 210 the same confining pressure. This phenomenon can be attributed to the Klinkenberg effect. Gas 211 permeability is relatively sensitive to gas pressure at lower gas pressures (less than $1 \mathrm{MPa}$ ).

212 There is no significant difference in the gas pressure sensitivity of gas permeability under 213 different confining pressures. In the second cycle of loading and unloading, the effect of gas 214 pressure on permeability is largely similar to that of the first cycle. Furthermore, the gas 215 permeability at low pressure is more sensitive to the gas pressure. When the gas pressure 216 exceeds $2 \mathrm{MPa}$, the change in gas pressure has little effect on the gas permeability.

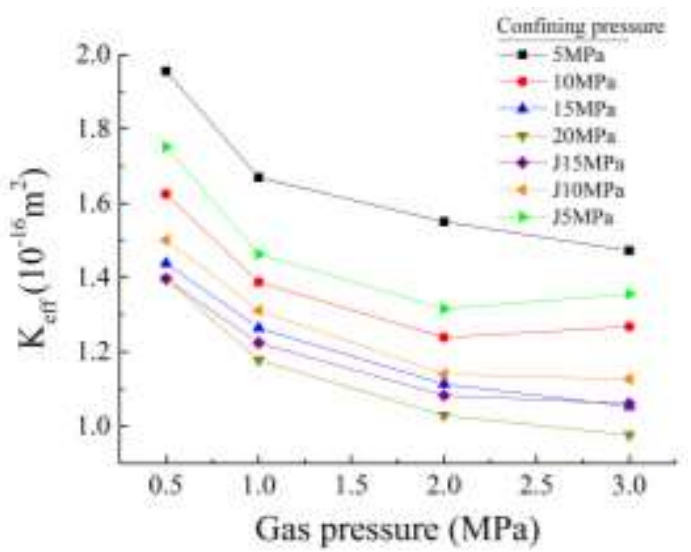

(a)

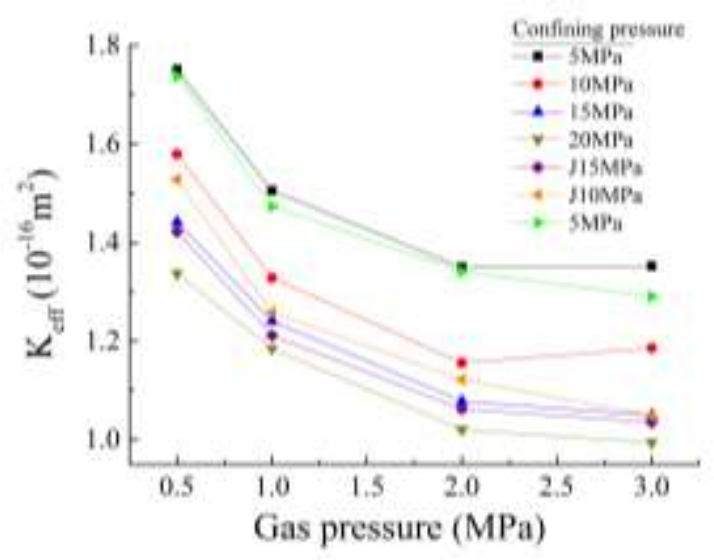

(b)

Figure 6. Effect of gas pressure on the gas permeability during (a) the $1^{\text {st }}$ loading-unloading phase; (b) the $2^{\text {nd }}$ loading-unloading phase.

Figure 7(a) gives an example of how to calculate the slip factor. For example, when $\mathrm{P}_{\mathrm{c}}=5 \mathrm{MPa}$, the fitting equation is $y=1.1+0.23 x$, and the intrinsic permeability is $1.1 \times 10^{-16} \mathrm{~m}^{2}\left(k_{\text {int }}\right)$. The slip factor can be obtained from $0.23 / 1.1=0.21$ (MPa). As a result, we can obtain the relationship between $k_{\text {int }}$ and $b$. Figure 7(b) illustrates the evolution trend of the slip factor and its relationship with inherent permeability (take sample $\mathrm{C} 1$ as an example). In general, the effect of slippage is more evident. There is a positive correlation between the two parameters (i.e., $l$ and $b$ ), whereas the pore radius is negatively correlated. From the mercury intrusion testmeasured pore size distribution (Figure 7(c)), it can be known that the median pore radius is about $0.129 \mu \mathrm{m}$, and the pores are mainly distributed between $0.01-1 \mu \mathrm{m}$. The smaller the pore size, the greater the slippage factor when the pore pressure remains constant.

The slip factor decreases as the intrinsic permeability increases, even though the data distribution is rather discrete. The pore size of the rock mass is correlated to this phenomenon. At constant temperature, the pore structure of the sandstone is compressed as the confining pressure increases. Extrusion of the pores results in a decrease in the pore size, leading to a 
decrease in the intrinsic permeability. The reduction in the pore size increases the frequency of collision between the gas molecules and the solid wall. As a result, the gas will slip flow, prompting the slip factor to rise. Previous research has also identified a similar phenomenon in gas permeability studies (Duan and Yang, 2014; Moghadam and Chalaturnyk, 2014; Tanikawa and Shimamoto, 2009; Yang et al., 2017).

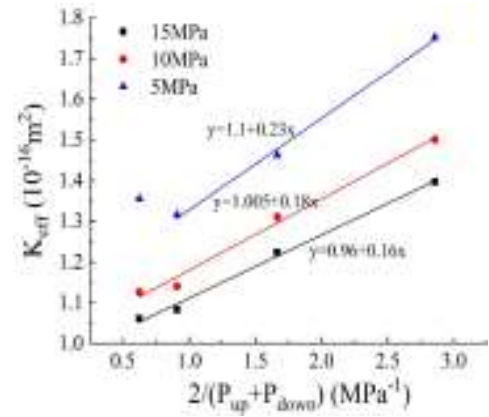

(a)

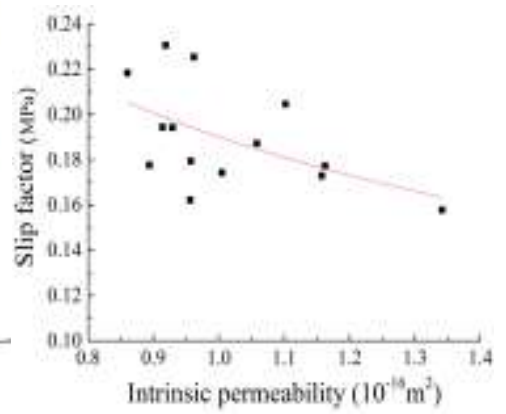

(b)

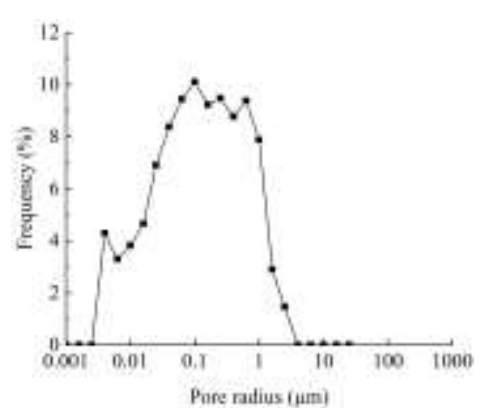

(c)

Figure 7. (a) An example of the slip factor calculation; (b) the relation between slip factor and intrinsic permeability; (c) the pore size distribution based on mercury intrusion test (Ni et al., 2021)

\subsection{Effect of loading pressure on gas permeability}

Figure 8 (a) shows the effect of axial pressure on gas permeability (take sample $\mathrm{C} 1$ as an example, confining pressure is constant). When the axial pressure is $5 \mathrm{MPa}$, the axial pressure has a negligible effect on the gas permeability. As axial pressure increases, the gas permeability increases mainly because the increase in the axial pressure causes the pores in the inner portion of the sample to open, increasing gas permeability. When the axial pressure is $30 \mathrm{MPa}$, the gas permeability reaches a maximum. When the axial pressure exceeds $30 \mathrm{MPa}$, as axial pressure rises, gas permeability decreases. In the experiments, when the axial pressure reached $30 \mathrm{MPa}$, the sample was fractured. At this time, the confining pressure has a compacting effect on the sample, resulting in a decrease in permeability. When the axial pressure is increased continuously, part of the crack gradually closes due to the axial pressure, resulting in a decrease

256 in permeability.

257 Figure 8(b) shows the deformation characteristics of the specimen during the application of the 258 axial pressure. Similar to the law of permeability change, the sample deforms slightly when the 259 confining pressure is less than $5 \mathrm{MPa}$. When the axial pressure is greater than $5 \mathrm{MPa}$, the 260 deformation of the sample gradually increases as the axial pressure increases. However, when 261 the axial pressure is greater than $20 \mathrm{MPa}$, the deformation of the sample decreases as the axial 262 pressure increases. This phenomenon is difficult to explain, and we will verify it in subsequent 263 trials. 


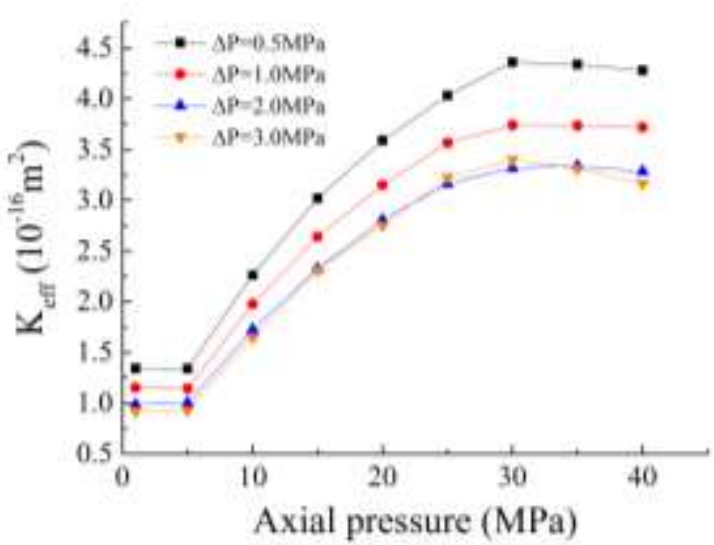

(a)

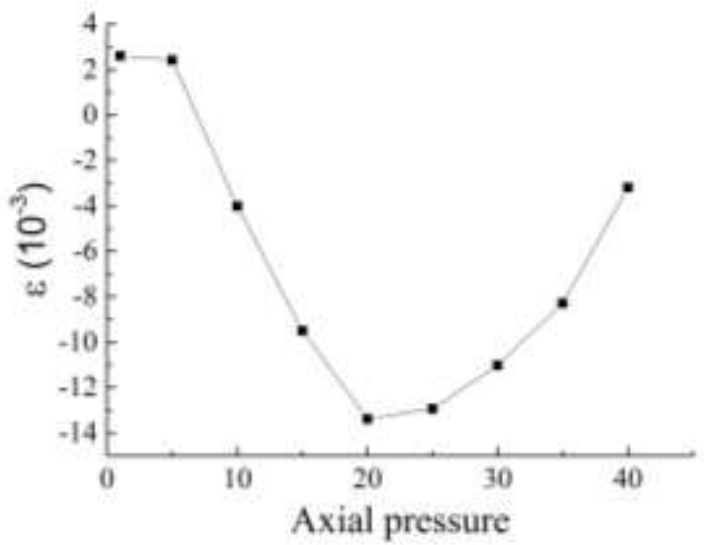

(b)

Figure 8. Effect of axial pressure on (a) gas permeability; (b) deformation.

\subsection{Micro-pore/fracture structure}

268 3.4.13D pore-fracture structure reconstruction

269 After the gas permeability test, sample C1 was scanned using a 3D X-ray Microanalyser. The 270 image resolution was $53.14 \mu \mathrm{m}$, which was scanned a layer at a time from top to bottom. After 271 the scanning was completed, a total of 500 slices were selected. Before reconstruction, image

272 enhancement and threshold segmentation are required. The whole process is shown in Figure

273 9. These slices were three-dimensionally reconstructed by a compiled algorithm to obtain a 274 three-dimensional reconstruction model of the sample.
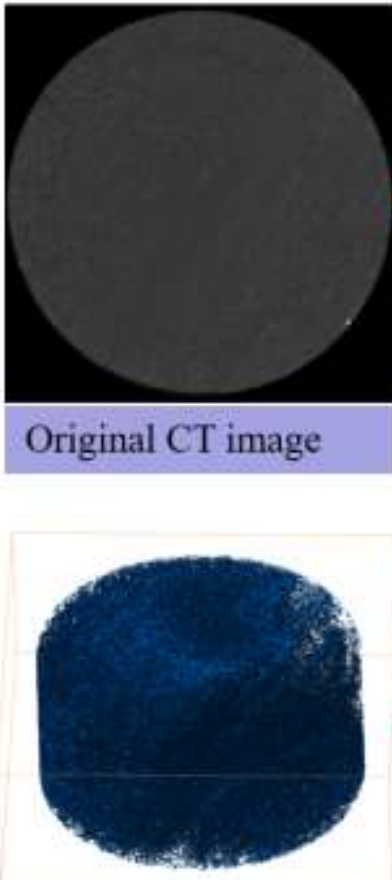

Pore

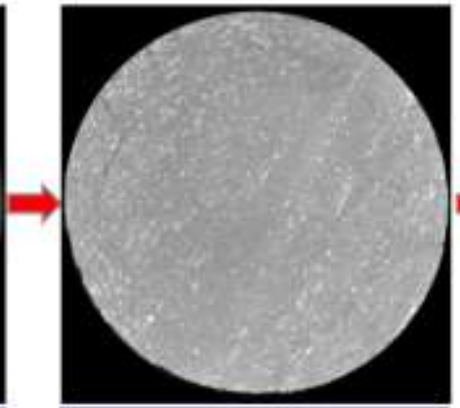

Image Enhancement, Nosie Redution

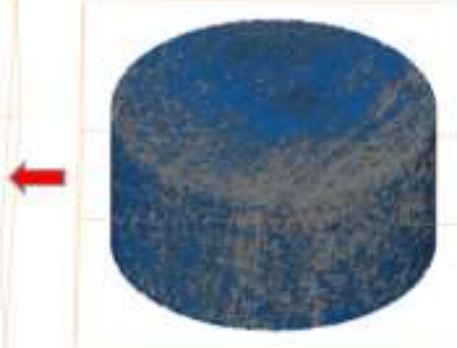

Matrix+pore

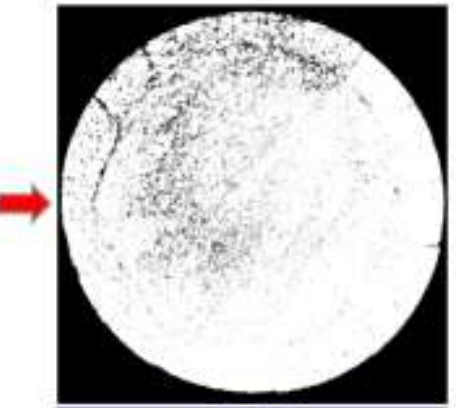

Threshold Segmentation

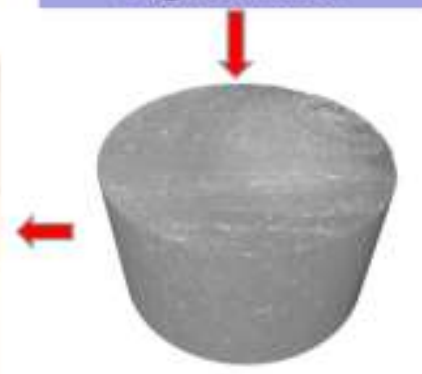

Reconstruction

Figure 9 Flow chart of 3D reconstruction of the digital core. 
As shown in Figure 10, there is a macroscopic crack in the height direction at an angle of

$27845^{\circ} \mathrm{C}+\frac{\varphi}{2}(\varphi$ is internal friction angle). CT scanning can identify rock fracture surfaces when 279 only macro cracks can be visible on the surface. This is primarily due to rock shear failure.

280 With the gradual increase in axial pressure in the static condition confining pressure, the friction 281 between the particles in the rock mass also gradually increases. When it reaches a certain value, 282 the rock mass particles will dislocate, and shear failure occurs. In addition to the shear fracture 283 surface, there are some small cracks.

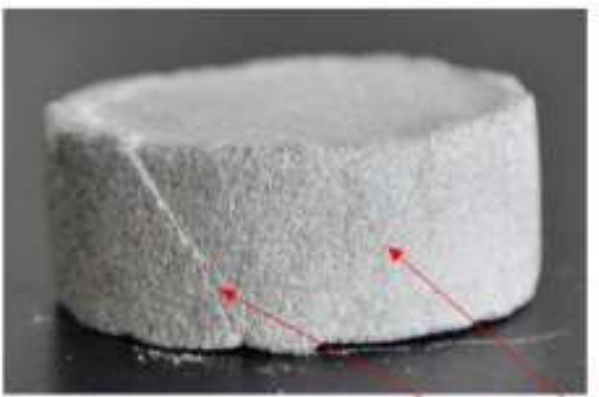

Sample after the triaxial test

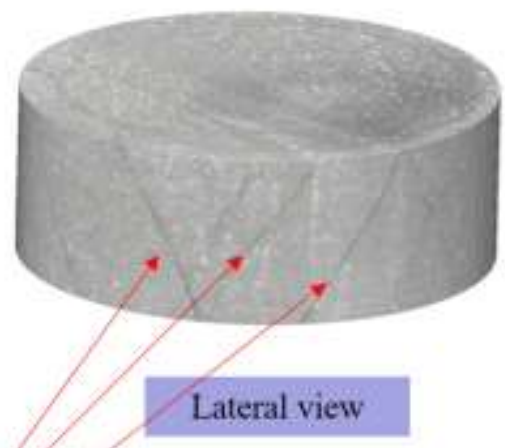

Lateral view

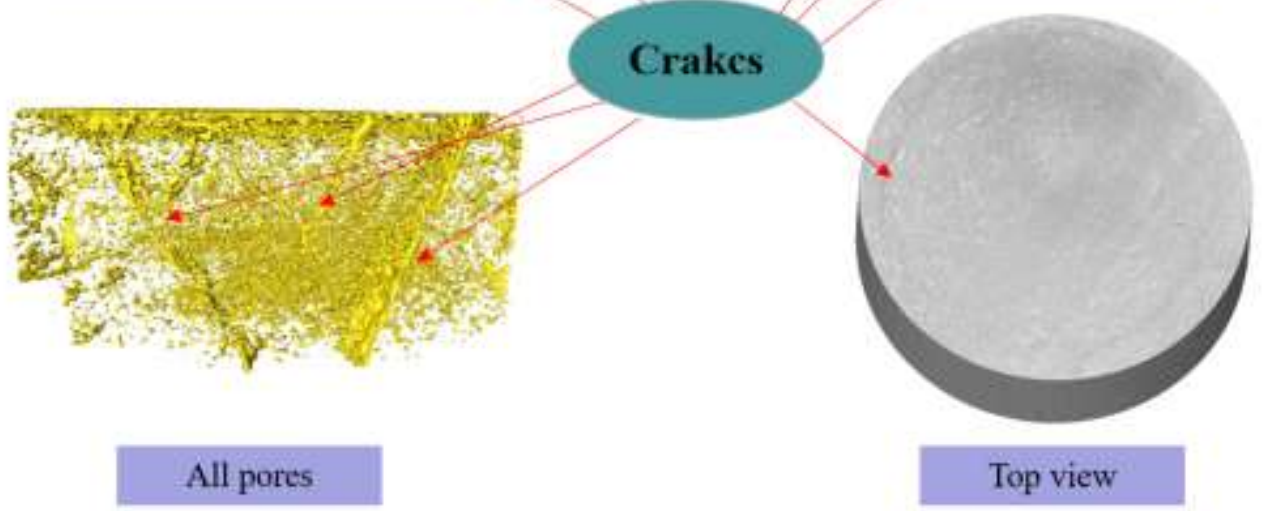

285 Figure $103 \mathrm{D}$ reconstruction model (3D X-ray) of sandstone after the end of the gas permeability test.

286 Figure 11 shows the evolution of the crack generation and permeability. Phase I: in the initial stage, the stress on the pore structure of the sample is weak due to the small axial pressure. Therefore, the permeability remains largely unchanged. Phase II: as the axial pressure increases, the micro-crack appears in the sample, and the gas permeability increases gradually. When a crack (shear failure) is generated at an angle of $45^{\circ} \mathrm{C} \pm \frac{\varphi}{2}$ degree in the height

291 direction, the gas permeability is maximized. Phase III: as the axial pressure increases, the axial 292 pressure will compress the crack, decreasing permeability. Therefore, the evolution of 293 permeability during the complete process varies as follows: stable $\rightarrow$ slowly increasing $\rightarrow$ 294 reaching peak $\rightarrow$ gradually decreasing. 


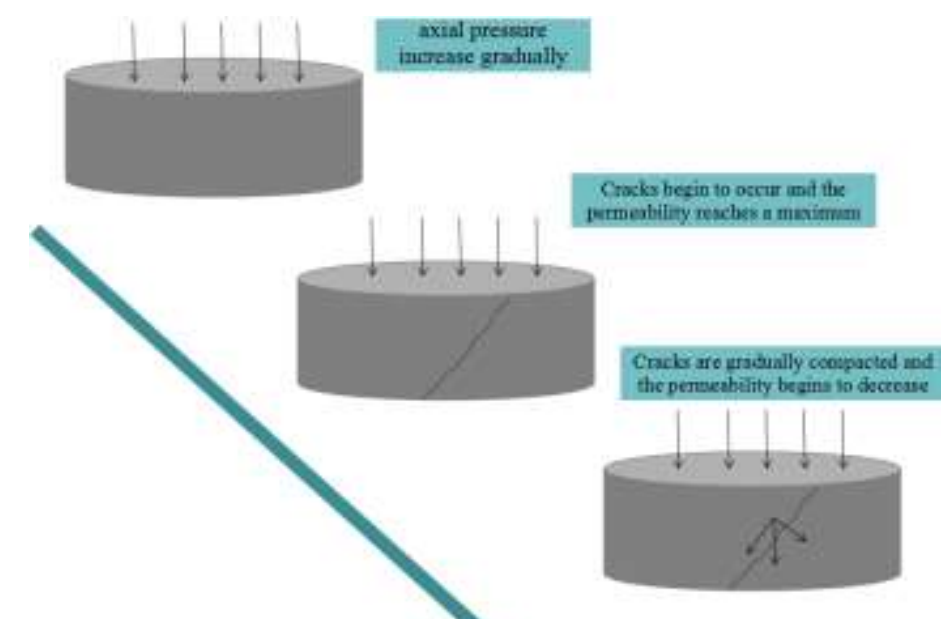

$$
L_{C}=\frac{V_{C}}{V_{T}}
$$

318 Where $L_{C}$ is the pore connectivity, $V_{C}$ is the total volume of the connected pores, and $V_{T}$ is 319 the total pore volume. The calculated pore connectivity of the sample is $64.41 \%$. According to 320 Figure.12, most connected pores are concentrated in half of the sample, while the isolated pores 321 mainly exist in the upper left part. Therefore, the main migration path of gas during gas flow

Figure 11. Schematic diagram of the crack development and permeability evolution under triaxial conditions.

3.4.2 Quantitative characterization of pore (crack) structure

To quantitatively characterize the pore structure of the sample, we separated the pore structure from the matrix. In the characterisation process, we found that the threshold algorithm has a greater impact on the characterization results. According to the suggestions of Liu et al.(2020), we make a comparative analysis of different algorithms. Figure 12 shows the calculation results based on the two thresholds. It can be found that when the threshold is 49 , the porosity is only $1.85 \%$. When the threshold is 51 , it is relatively close to the calculated result of the physical experiment. The porosity was calculated as $7.28 \%$ by subsequent processing of the CT image. This result is less than the value (10\%) measured by the nitrogen adsorption test due to the CT's resolution not allowing some of the smaller pores to be seen. For some nanopores, higher resolution images are needed to characterize them. Usually, the image scanning accuracy is related to the sample size, and a higher resolution digital image can be obtained from a small sample. For nanopores, FIB/SEM (Focus Ion Beam Scanning Electron Microscopes) is a better choice, and the effect is better. This part of the research aims to observe macroscopic cracks, so no higher resolution image scanning is performed.

Because the isolated pore fracture does not contribute to the seepage characteristics, we extract the connected pores from all pores and calculate the pore connectivity:

should be via macroscopic fractures and connected pores. 


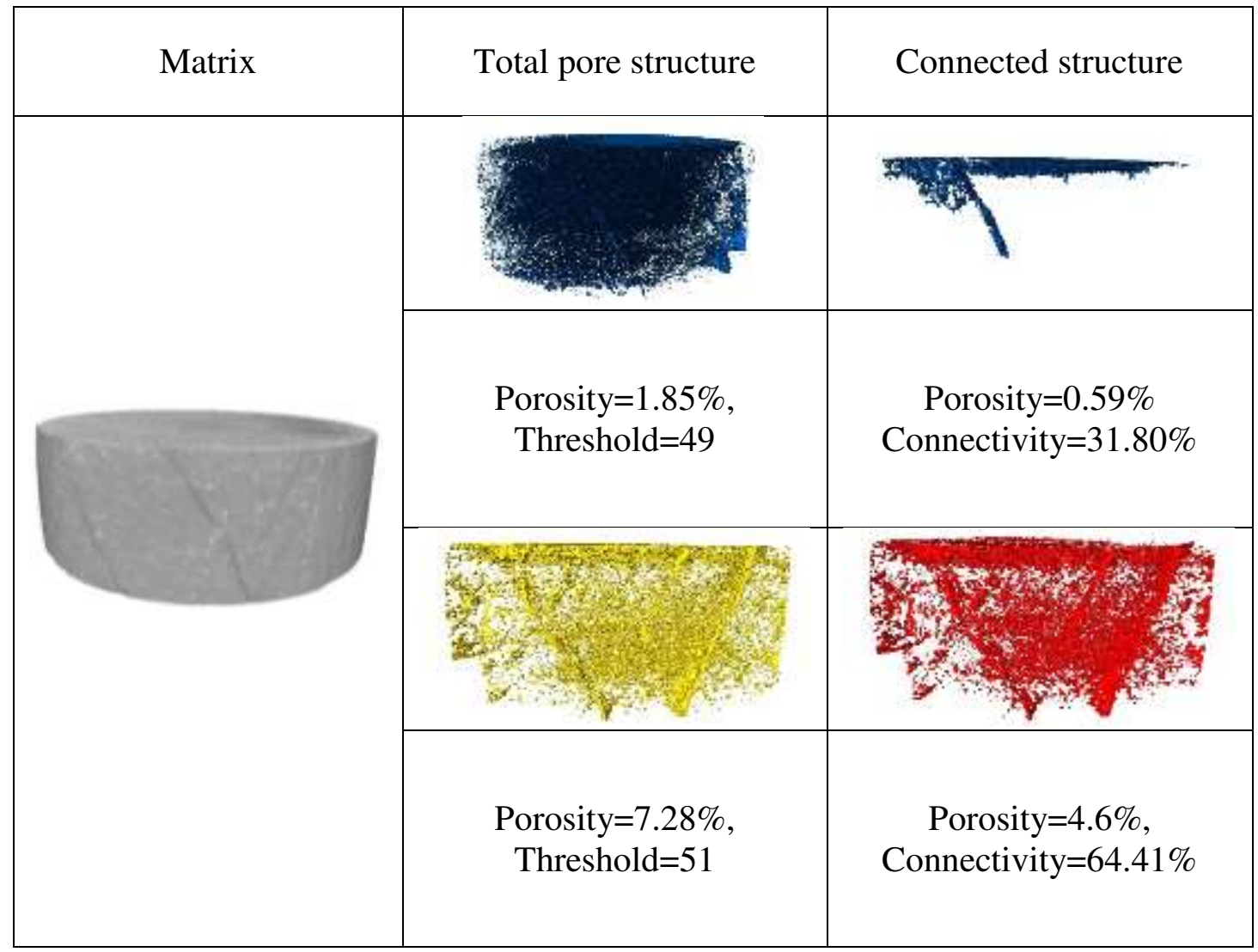

Figure 12. Pore network and interconnected pore network of sandstone

\section{Conclusions}

325 This study systematically examined the gas permeability of two sandstone samples under various conditions, including confining pressure, axial pressure, and gas pressure. First, the influence of cyclic loading and unloading on the evolution of gas permeation was tested. The evolution of the gas permeability occurred primarily in the first cycle of loading and unloading. Especially at the initial loading stage (confining pressure less than $5 \mathrm{MPa}$ ), the gas permeability decreased by 7.59 and 2.27 times for samples $\mathrm{C} 1$ and $\mathrm{C} 2$, respectively. From $5 \mathrm{MPa}$ to $20 \mathrm{MPa}$,

331 the gas permeability decreased only a little. This means that most of the pores in the initial stage that allow the gas to flow have been closed, and subsequent pressurization has less effect on the deformation of the pore structure. After two loading and unloading cycles, the gas permeability recovered to $4.07 \times 10^{-16} \mathrm{~m}^{2}(\mathrm{C} 1)$ and $2.51 \times 10^{-16} \mathrm{~m}^{2}(\mathrm{C} 2)$, which is fairly different from the initial value. This finding indicates that some of the pores have been plastically deformed and cannot be recovered.

337 When axial pressure is applied, the gas permeability gradually increases due to the expansion 338 of the crack. When macroscopic cracks are generated, the permeability reaches a maximum.

339 The axial pressure increased further, and the permeability reduced due to the compaction of the axial pressure on the macro crack. Based on microscopic CT images, the cracks are mainly

341 oriented at a $45^{\circ} \mathrm{C}$-degree angle along the height direction. Further quantitative analysis 
revealed that the porosity was approximately $7.28 \%$, which was relatively tight and indirectly

343 verified the phenomenon of low macroscopic gas permeability. In addition, different algorithms

344 have a greater impact on digital image characterization, and further research is needed.

345 In addition, we also considered the Klinkenberg (gas slippage) effect and found that when the 346 gas pressure exceeds $2 \mathrm{MPa}$, the change in gas pressure has little effect on the permeability.

347 There is no significant difference in the gas slippage effect under different confining pressures, 348 and the slippage effect in the loading stage is clearer than that in the unloading stage. Studies 349 have also found that the slip factor tends to increase as the intrinsic permeability decreases.

350 This phenomenon can be primarily attributed to the pore structure being subjected to pressure 351 and causing the pore size to decrease. As a result of this increased collision frequency between 352 the gas molecules and the inner wall of the pores, the gas exhibits a slip phenomenon and 353 increasing the slip factor.

\section{Acknowledgment}

355 The authors are grateful for the support of the National Natural Science Foundation of China 356 (No. 51809263; 52174133), China Postdoctoral Science Foundation (2019M661993), and the 357 Open Fund of MOE Key Laboratory of Deep Earth Science and Engineering (Sichuan 358 University) (Grant No. DESE201906). The scanning service supported by Advanced Analysis $359 \&$ Computation Center, China University of Mining and Technology is also greatly 360 acknowledged.

361 Declarations

362 Conflict of interest The authors declare that they have no conflict of interest.

\section{References}

364 Amann Hildenbrand, A., Dietrichs, J.P., Krooss, B.M., 2016. Effective gas permeability of Tight Gas Sandstones as a function of capillary pressure - a non-steady-state approach. Geofluids 16, 367383.

Duan, Q.B., Yang, X.S., 2014. Experimental studies on gas and water permeability of fault rocks from the rupture of the 2008 Wenchuan earthquake, China. Science China Earth Sciences 57, 2825-2834.

Duan, Z., Davy, C.A., Agostini, F., Jeannin, L., Troadec, D., Skoczylas, F., 2014. Gas recovery potential of sandstones from tight gas reservoirs. International Journal of Rock Mechanics \& Mining Sciences 65, 75-85.

Fu, X., Agostini, F., Skoczylas, F., Jeannin, L., 2015. Experimental study of the stress dependence of the absolute and relative permeabilities of some tight gas sandstones. International Journal of Rock Mechanics \& Mining Sciences 77, 36-43.

Ghanbarian, B., Torresverdín, C., Skaggs, T.H., 2016. Quantifying tight-gas sandstone permeability via critical path analysis. Advances in Water Resources 92, 316-322.

Khlaifat, A., Qutob, H., Barakat, N., 2011. Tight gas sands development is critical to future world energy resources, Society of Petroleum Engineers.

Klinkenberg L J. The Permeability of Porous Media To Liquids And Gases. Socar Proceedings, 1941, 2(2):200-213.

Liu, J.-F., Ni, H.-Y., Cao, X.-L., Ma, L.-K., Guo, J.-N., Chen, X., 2020. Laboratory investigation on gas permeability of compacted GMZ bentonite under a coupled hydraulic-mechanical effect. Engineering Geology 276, 105761. 
Liu, J.F., Skoczylas, F., Talandier, J., 2015. Gas permeability of a compacted bentonite-sand mixture: coupled effects of water content, dry density, and confining pressure. Canadian Geotechnical Journal 52, 1159-1167.

Liu, J.F., Cao, X.L., Xu, J., Yao, Q.L., Ni, H.Y., 2020. A new method for threshold determination of gray image. Geomechanics and Geophysics for Geo-Energy and GeoResources, 6(4):72.

Moghadam, A.A., Chalaturnyk, R., 2014. Expansion of the Klinkenberg's slippage equation to low permeability porous media. International Journal of Coal Geology 123, 2-9.

Ni, H., Liu, J., Huang, B., Pu, H., Meng, Q., Wang, Y., \& Sha, Z.,2021. Quantitative analysis of pore structure and permeability characteristics of sandstone using SEM and CT images. Journal of Natural Gas Science \& Engineering, 88, 103861.

Tanikawa, W., Shimamoto, T., 2009. Comparison of Klinkenberg-corrected gas permeability and water permeability in sedimentary rocks. International Journal of Rock Mechanics \& Mining Sciences 46, 229-238.

Wang, H.L., Xu, W.Y., Cai, M., Xiang, Z.P., Kong, Q., 2017a. Gas Permeability and Porosity Evolution of a Porous Sandstone Under Repeated Loading and Unloading Conditions. Rock Mechanics \& Rock Engineering 50, 1-13.

Wang, Y., Agostini, F., Skoczylas, F., Jeannin, L., Portier, É., 2017b. Experimental study of the gas permeability and bulk modulus of tight sandstone and changes in its pore structure. International Journal of Rock Mechanics \& Mining Sciences 91, 203-209.

Wang FY, Jiao L, Lian PQ, Zeng JH. 2018. Apparent gas permeability, intrinsic permeability and liquid permeability of fractal porous media: Carbonate rock study with experiments and mathematical modelling. Journal of Petroleum Science and Engineering, 173, 1304-1315.

Wang, F, Cheng, H., 2020. Effect of tortuosity on the stress-dependent permeability of tight sandstones: Analytical modelling and experimentation. Marine and Petroleum Geology. 120: 104524.

Wu, T., Pan ZJ., Connell LD., Cameilleri M., Fu XF. 2020. Apparent gas permeability behaviour in the near critical region for real gases[J]. Journal of Natural Gas Science and Engineering, 77.

Xiao, D., Lu, S., Yang, J., Zhang, L., Li, B., 2017. Classifying Multiscale Pores and Investigating Their Relationship with Porosity and Permeability in Tight Sandstone Gas Reservoirs. Energy \& Fuels 31, 9188-9200.

Yang, D., Wang, W., Chen, W., Wang, S., Wang, X., 2017. Experimental investigation on the coupled effect of effective stress and gas slippage on the permeability of shale. Scientific Reports 7, 44696.

Yang, S.Q., Huang, Y.H., 2020. Effect of damage on gas seepage behavior of sandstone specimens. Journal of Rock Mechanics and Geotechnical Engineering 12, 866-876.

Zhang, H., Zhong, Y., Kuru, E., Kuang, J., She, J., 2019. Impacts of permeability stress sensitivity and aqueous phase trapping on the tight sandstone gas well productivity - A case study of the Daniudi gas field. Journal of Petroleum Science and Engineering 177, 261-269.

Zhang, Y., Wang, L., Li, H., Zhang, Y., Fu G. Experimental study of the permeability of fractured sandstone under complex stress paths. Energy Science \& Engineering 8(9): 3217-3227. 